\title{
Use of hyaluronic acid filler and botulinum toxin type $A$ in facial renjuvenation
}

\author{
T Vitagliano ${ }^{2 *}$, U Bottoni ${ }^{1}$, A Greto Ciriaco ${ }^{2}$, D Novembre ${ }^{2}$, M Greco $^{2}$ \\ From de Senectute: Age and Health Forum \\ Catanzaro, Italy. 5-7 December 2009
}

\begin{abstract}
Background
Facial soft tissue augmentation and rejuvenation have become over the past several years the practice more requested in aesthetic medicine. The authors share their experiences to evaluate long term safety, clinical effectiveness and patient satisfaction regarding the injection of hyaluronic acid (HA) filler and botulinum toxyn tipe A (BTTA). In some cases the patient received a combined treatment of HA and BTTA to obtain absolute aestethic satisfaction.
\end{abstract}

\section{Materials and methods}

From June 2007 to June 2009, a total of 208 caucasian patients (179 female and 29 male) received a HA or BTTA or combined (63 cases) injection in differents facial areas: oral commissure, nasolabial folds 1, lips, cheeks, chin. Formulations used in this study are the $20 \mathrm{mg} / \mathrm{mL}$ HA smooth, highly cohesive, viscous, fully reversible, volumizing filler 2 indicated to restore facial volume and 50 units of Clostridium botulinum type A neurotoxin complex, added with $1,0 \mathrm{~mL}$ of saline solution $0,9 \%$. This trial has been structured with a preliminary visit to evaluate the loss of volume and the individual expectations. All injections of HA were in the deep dermis or above the periosteum whereas BTTA were performed in intramuscular site in frontal, orbitozygomatic regions and in glabellar area. The most part of injections were not performed under local anesthesia and the mean total of injection volume per each patient was about $4.4 \mathrm{~mL}$ for $\mathrm{HA}$ and $1,0 \mathrm{~mL}$ for BTTA 3 . Every patient was photographed at each visit, before and after the treatment with a follow up at 7, 14, 21 and 45 days, recording procedural details, effectiveness, aesthetic outcomes and adverse events. Patients were also asked to evaluate their personal satisfaction by using a

2Department of Plastic Surgery, University "Magna Graecia", Catanzaro, Italy subjective scale from 0 (no satisfaction) to 5 (absolute satisfaction) starting from pre treatment photos. Finally they were asked if they would repeat the treatment again.

\section{Results}

Volume loss declined significatly after treatment and every patient noted a positive change in his own appearance. Local adverse events appeared only in three patients: two of those consisting in a local transitory edema and inflammatory reaction and the third was a slight blepharoptosis for BTTA, normalized one month later.. Personal satisfaciotn has been absolute in 182 patients $(87.5 \%)$ and $98 \%$ of the patients would repeat treatment.

\section{Conclusions}

The $20-\mathrm{mg} / \mathrm{mL}$ smooth, highly cohesive, viscous, volumizing HA filler and the BTTA were effective, well tolerated and easy to use in clinical practice.

\section{Author details}

'Department of Dermatology, University "Magna Graecia", Catanzaro, Italy.

2Department of Plastic Surgery, University "Magna Graecia", Catanzaro, Italy.

Published: 19 May 2010

\section{References}

1. Onesti M, Toscani M, Curinga G, Chiummariello S, Scuderi N: Assessment of a new hyaluronic acid filler. double-blind, randomized, comparative study between Puragen and Captique in the treatment of nasolabial folds. In Vivo 2009, 23(3):479-486.

2. Bergeret-Galley C: Choosing injectable implants according to treatment area: the European experience. Facial Plast Surg 2009, 25(2):135-142.

3. Gadhia K, Walmsley AD: Facial aesthetics: is botulinum toxin treatment effective and safe? A systematic review of randomised controlled trials. Br Dent J. 2009, 207(5):E9 216-217.

doi:10.1186/1471-2318-10-S1-A82

Cite this article as: Vitagliano et al:: Use of hyaluronic acid filler and

botulinum toxin type A in facial renjuvenation. BMC Geriatrics 2010

10(Suppl 1):A82. 